\title{
COVID-19: A Physical Model
}

\author{
Kai W. Wong1, Peter C. W. Fung2, Wan K. Chow ${ }^{3}$ \\ ${ }^{1}$ Department of Physics and Astronomy, University of Kansas, Lawrence, USA \\ ${ }^{2}$ Department of Physics, Department of Medicine, and Centre on Behavioral Health, University of Hong Kong, Hong Kong, China \\ ${ }^{3}$ Department of Building Services Engineering, The Hong Kong Polytechnic University, Hong Kong, China \\ Email:kww88ng@gmail.com
}

How to cite this paper: Wong, K.W., Fung, P.C.W. and Chow, W.K. (2020) COVID-19: A Physical Model. Open Journal of Biophysics, 10, 88-95.

https://doi.org/10.4236/ojbiphy.2020.102008

Received: March 21, 2020

Accepted: April 11, 2020

Published: April 14, 2020

Copyright (C) 2020 by author(s) and Scientific Research Publishing Inc. This work is licensed under the Creative Commons Attribution International License (CC BY 4.0).

http://creativecommons.org/licenses/by/4.0/ (c) (i) Open Access

\begin{abstract}
The coronavirus structure is explained as a fractal representation of the Poincare sphere of proteins, dotted with t-spikes having the geometric realization of the $\mathrm{SU}$ (3) Lie group for the protein spike, and encompasses an RNA of $S U(2)$ topologies representations, while within the core contains a virus DNA. Through this suggested model, the virus must possess a critical temperature $T_{c}$ induced by the EEM mechanism for the completion of the Poincare sphere surface. Thus above $T_{c}$ it will disintegrate. We then discuss how the virus transmission progresses within the patient's body, and explain a very fast recent detection method currently used consistent with this model, as well as a corresponding possible cure based on this same principle of the body's immune system. Hopefully the model can be also used as a guide to finding possible medications, so far is lacking.
\end{abstract}

\section{Keywords}

COVID-19, Perelman Mappings, Virus Survival Temperature $T$, Virus Multiplication Mechanism

\section{Introduction and the Physical Model}

The recent coronavirus pandemic has created great economic damages to the world on top of the health pandemic. On the other side, because of this disaster we have learned a lot about the structure and progress and multiplication mechanisms of the virus in the human body. We shall try to explain below how we can apply physical principles derived from Perelman mappings of the homogeneous 5D space-time into its physical shape and summarize this deadly COVID-19 operation.

All coronaviruses are basically spherical in shape, covered with " $t$ " shape spikes on the surface. Each virus spike contains a unique RNA within. And each 
spike is a protein composed of 3 vertical parallel strains, displaying the $\mathrm{SU}(3)$ fractal representation of the proton/neutron formation from the quark model, and enclosing a linear void in the center, clearly showing the potential of housing an RNA structure. The spherical surface is a lipid bilayer on which many proteins are inserted. Some of these proteins have very strong "intruding power" so that the virus lets itself be engulfed by many types of living cells when in contact. The virus attacks not only respiratory cells, but can get into gastric cells, or even neurons. It cannot enter the skin which is protected by the epithelium. In terms of 5D projection into the Lorentz $4 \mathrm{D}$ manifold physical principles, it implies the virus is formed through the two steps of Perelman mappings, such that its topological surface must repeat the SU(3) and SU(2) Lie group symmetry [1], by gathering the proteins within the surrounding thermal bath, as induced by the DNA spectrum, which is the eigen-solutions of the Maxwell DLRO (Diagonal Long Range Order) monopole loop created via thermal energy supplied to the DNA, as explained by us earlier [2], while according to the Excitonic Enhancement mechanism model [3], the formation of the spherical protein coating requires the temperature below a control value $T_{c}$ of the ODLRO (Off-diagonal Long Range Order) on the Valence band "p" electron hole orbitals [4]. Hence, we expect the surface to be dotted with t-spikes, having structure of 3 gathered in the corners of an equilateral triangle, representing $\mathrm{SU}(3)$ topology, while a 1-dimensional monopole 2ec expanding boson field composed of e, -e massless spinor pair if allowed, would be along the RNA, except due to the finite fixed length of the RNA it does not exist as explained in ref. [2] through the center of the triangle, thus obeying $\mathrm{SU}(2)$. (See Figure 1 for illustration of the virus.)

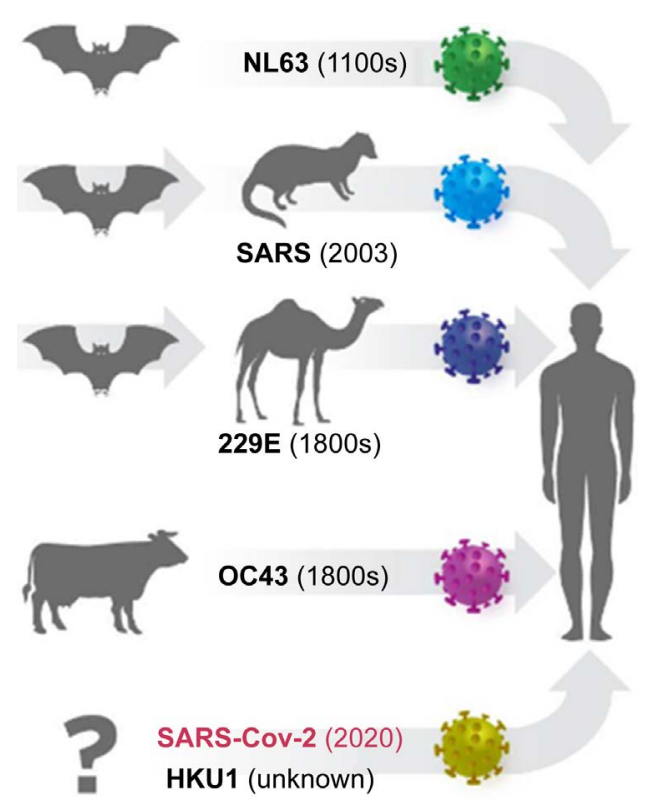

Figure 1. Epidemic potential. (Source: Timothy Sheahan, University of North Carolina. In: B. McKay and T. Ansari, "Coronavirus Symptoms and How to Protect Yourself: What We Know”, The Wall Street Journal, 19 March 2020.

https:/www.wsj.com/articles/what-we-know-about-the-coronavirus-11579716128) 
Because such a structure is the simplest fractal topological structure that we can create out of Perelman mappings into a Poincare sphere due to the necessary realization in the $4 \mathrm{D}$ Lorentz space-time from the homogeneous 5D mapping. The varying sizes and varying number of spikes make the different Coronaviruses. The monopole eigen-state within the DNA energy must be maintained via chemical decomposition of matters in the surrounding via oxidation or external optical source, such as thermal or light sources. Without the energy, the monopole will decay, and the virus will also decompose, releasing the RNAs in the " $\mathrm{t}$ " spikes. For each pair of such RNAs, when energy is available, the nitrogenous end caps Uracil will be replaced by the Thymine in the DNA. To achieve this, the loss spikes must be attached first to the patient's oxidation energy source, so that the released RNA can form new DNA. Then through the Perelman 3D entropy manifold mapping the new virus can be created. This is the main reason why the coronavirus must attach itself to the respiratory path wall. Furthermore, as that happens that when a new virus can be born, the temperature in the surrounding must increase, but it must remain not to exceed $T_{c}$ for the shell formation: This is a very delicate constrain.

To understand quantitatively, let us review the Excitonic Enhancement Mechanism model [3] that was proposed for the HTC High Temperature Cuprates Superconductors [5], where the ODLRO of the VB valence bond ' $\mathrm{p}$ ' holes orbitals were induced via the formation of excitons between the Fermi level and the $\mathrm{CB}$ conduction band and its coupling to the holes in place of the phonons in the BCS superconductor mechanism. By extending this model for the natural formation of bio cells within the human body, we can provide an estimation on the $T_{c}$ in normal human cells.

According to the $5 \mathrm{D}$ model, the cell growth is caused by the induced ODLRO in the VB " $p$ " hole orbitals, which comes from the periodic Oxygen in the $\mathrm{CuO}$ plane for the culprate superconductors, and the Carbon in the periodic spherical surface protein shell for the bio cells, and similarly the coronavirus, via the EEM mechanism.

The normal body temperature is roughly $100^{\circ} \mathrm{F}$, or $36^{\circ} \mathrm{C}$, which corresponds to $309^{\circ} \mathrm{K}$. In order for the normal growth $T_{c}$ must be $>$ than this temperature. Let us assume then $T_{c}=311^{\circ} \mathrm{K}$.

The EEM superconducting gap $\Delta_{G}[6]$ is given by

$$
\Delta_{G}=\left\{\Delta_{B C S}^{2}+\Delta_{e x}^{4} /\left[e_{o}+\mu+G\right]^{2}\right\}^{0.5}
$$

[see Wong and Ching papers on the derivation of Equation (1) in the EEM theory]

$\Delta_{\text {ex }}$ is the exciton binding, and $<\mu+G, G$ is the positive bandgap, while $\mu$ is the Fermi level of the holes. $\varepsilon_{o}$ is the correction due to the presence of excitons.

Using the Boltzmann constant $k_{B}=1.38 \times 10^{-23} \mathrm{~J} /{ }^{\circ} \mathrm{K}$.

By ignoring $\Delta_{B C S}$ and $\varepsilon_{o}$, we obtain a numerical result

$$
\mu+G \text { of } 2 \mathrm{eV}
$$


and $\Delta_{\text {ex }}$ of $1 \mathrm{eV}$. that would satisfy a $T_{c}$ of $311^{\circ} \mathrm{K}$.

For HTS culprates $\Delta_{\mathrm{ex}}$ is roughly $0.5-0.6 \mathrm{eV}$, while the rest of the electron band structure parameters $\mu$ and $G$ are similar. We then expect $T_{c}$ to be no more than $120^{\circ} \mathrm{K}$. The enhanced $T_{c}$ in bio cells must be much higher, in fact higher than the body normal temperature, due essentially to the exciton effective mass being smaller.

This quantitative analysis is equally applicable for estimating the Coronaviruses $T_{c}$ In fact it must lie above the body cells $T_{\mathcal{c}}$, but less than the max fever $T$ before the onset of pneumonia, which can be set as $20^{\circ} \mathrm{K}$ higher. Hence the $T_{c}$ for the Coronaviruses which covers from the common flu to SARS and MERS, must be between $311^{\circ} \mathrm{K}$ to $331^{\circ} \mathrm{K}$.

As the body $T$ raises close to $T_{c}$ of the virus, the virus multiplication slows, due primarily to the EEM induced broadening of the HTC transition. Thus the most active $T$ range of the virus must be between the body's normal $T$ and its $T_{c}$ value. Suppose the COVID-19 $T_{c}=330^{\circ} \mathrm{K}$, and is most active around the body $T$, since the reproduction probability follows an exponential curve, which governs the rate of transmission within a human population. This works out to $36^{\circ} \mathrm{C}$. As temperature raises above $36^{\circ} \mathrm{C}$, the virus reproduction decreases rapidly.

The higher the shell formation $T_{c}$, the more deadly it would be, because it can multiply easier. However it also implies the monopole eigen-energy requirement is also higher, which in turn due to the uncertainty principle $\mathrm{d} E \mathrm{~d} t \geq h$, tends to shorten the life time of the virus which is proportional to $\mathrm{d} t$, thus reduces its effective ability to transmit when away from the human body. As such, due to the environment content of proteins available, and the $T$ value reachable, the virus for its own survival constantly makes minor changes, very much like the common flu viruses.

As shown in Figure 1, most Coronaviruses are carried by bats, where the viruses must be harmless, means the bats body immune and protein composition does not favor the multiplication of the virus. This same principle probably explains why COVID-19 is less deadly in kids than adults. A better quantitative research on the general protein compositional changes with our body aging could be helpful in our search of a prevention.

\section{Mechanism of Propagation of COVID-19 within the Patient}

It is due to the above explanation, the first stage symptom of the COVID-19 in a patient is always fever [7] as new viruses are formed from the spikes penetrating into a body's cell so that the RNA length can be extended temporarily to accommodate the creation of a 2ec monopole boson, accompanied with the oxidation of the proteins on the virus shell, which in turn breaks the virus shell so as to be able to change the RNA end caps from Uracil to the Thymine and created the DNA, from which new coronavirus can be formed around it as we discussed in the previous section. Hence it is most efficient for the virus to multiply along the respiratory track. This $T$ raises coincide with inducing the body's immune 
response, which operates as $T$ rises, and in turn triggers the white blood $\mathrm{T}$ cells to seek and consume the virus. Thus young healthy people usually can recover as the white blood $\mathrm{T}$ cells win over the limited number of new rebirth of the viruses, due primarily from limited proteins available caused by the lack of extra sugars and fat proteins generally present in older people. At this stage, when many new viruses are created from the dead ones left over for a patient in the respiratory track are sometimes accompanied with dry cough as the body's respiratory expels the dead virus that did not make it revived by forming new viruses. With that life viruses are also ejected, thus spreading the virus transmission in droplets covering the near surrounding. Hence to slow down the direct person to person transmission of the virus, social distancing, frequent sensitizing including hands, patient isolation, and population mobility confinement is of paramount importance. Disposed dead virus must remain within the patient's blood for some time, as the immune mechanism that destroys the virus is through the T-cells, which of course is carried through the body by the blood flow. Hence, it is easy to detect the Coronavirus infected patient via blood test for the presence of the virus DNA, even when the patient had totally recovered. This technique of testing has in fact been created and applied by the company Biomerica, as a demonstration of its erology patented method. The test is simple and the result is $100 \%$ and very quick, except it gives false positive if the patient already recovered. Hence the positive test must be simultaneously verified by direct detection of the virus present in the mucus obtained from the throat, plus no lung cell damage using X-ray photographs or CT scan. The coronavirus detection must then always be simultaneously cross verified with all three detection methods. When the immune response fails for the first stage, the virus will find its path to enter the lung. At this final stage, the virus is attached to the lung cells. The death of a virus, will produce mucus which is the waste left from the protein broken shell, which in turn clog up the effective average air cell volume, thus changing through the general gas law: $P V I T=$ constant, where $P$ is the air pressure within the lung cells, $V$ is the effective lung cell column. Thus the reduction of $V$ lowers the $T$ in the lung. A severe $T$ drop below $T_{c}$ leads to even more virus creation ending in Pneumonia for the patient, and high fever returns again, even with $T>$ $T_{c}$ But before that, the patient can lose the battle for life, before the virus is destroyed by the breaking of the ODLRO transition as the fever's $T$ exceeds $T_{\mathcal{O}}$ causing the virus self-reduction. Hence, at this last stage, buying time is essential. The only method then is to keep the effective air and oxygen volume in the lung as long as possible. Unfortunately, even if the patient recovers from this stage, his or her lung cells might be permanently compromised, leaving a lifelong damage.

\section{Conclusion and Some Suggestions for the COVID-19 Medication}

We look at the HTC crystals. That is formed by simple phase transition from 
liquid to crystal solid, which produces the band structure, with periodic boundary condition. But bio cells do not go through such a phase transition, it is induced by a DLRO within itself. That requires the presence of a DNA, which can have a gene spectrum from the monopole boson DLRO state. Hence the virus must be introduced into a patient from outside. It cannot self-develop within the body. But even with the presence of a virus DNA, that is not enough. The presence of proteins meaning carbon based flexible structures such as ACE2 must be present in virus surrounding thermal bath, then through Perelman entropy mappings it can form the Poincare sphere, which gives the periodic boundary, such that an ODLRO can exist in the electronic orbitals of the shell via EEM to satisfy the LRO matching. This requires also the thermal bath $T<T_{c}$. Thus DNA must always exist first in every process. Now considering $T_{c}$ specific to the Coronavirus. To form the DNA, out of just RNAs, is not possible. It needs the original introduction of such a virus DNA into the thermal bath. Then the virus can be generated. In fact, it can multiply, only when RNAs are also present, like human reproduction allowing the genome to track the heritage of the virus [8]. Of all the 9 known Coronaviruses, only 3, that are SARS, MERS and COVID-19 shown to be deadly, the remainders act much like the common flu. Hence we have new DNAs, again all due to DLRO inducing global order. But that needs energy similar to crystal phase transition. Hence there is another $T(o)$ for this to happen. This $T(o)$ needs not to be $<$ or $>$ than $T_{\mathrm{c}}$ for the EEM $T_{c}$. The lower $T(o)$ the faster the virus can multiply when one breaks up. The break up comes from multiple possibilities. First, the DNA monopole eigenstate can lose energy to the thermal bath, due to the $h v$ spectra, the high $v$ frequency will lose energy just via conductivity, or $T$ raises to $T_{c}$. Hence the virus has to obtain energy input, via extra energy obtained via oxidation in immediate contact. It is this that drives the virus to multiply and survive, and the reason the virus tries to attach itself to the respiratory path, where most oxidation process happens. The more RNAs the spikes carry the faster the virus could evolve, which might make it more or less transmittable, and might end up less deadly to human? Our aim then to eliminate the current COVID-19 pandemic must be a medication that will induce COVID-19 to mutate into a new less deadly form, and to find a vaccine that boosts our immune system to prevent the multiplication of the COVID-19 within our respiratory track.

Because the body's immune response must be accompanied by a temperature rise, it is therefore paramount that COVID-19 patients do not take fever reducing medications.

Through this analysis, we learn not just the progress of COVID-19, but also how simple life cell model like the coronavirus comes about, as well as the triggering of its necessary evolution. The other results we get from this study, allows us to model a prevention of its spread. Obviously, that is naturally accomplished by weather condition changes, through first and foremost the destruction of the monopole spectrum in the DNA, either by lack of energy supply, or by over 
supply via climate change. In another word COVID-19, is seasonal, similar to the flu virus. Secondly, as the COVID-19 virus changes, it might either increase or decrease its ability to transmit, as its survival time changes. Thirdly, once the body's immune system recognizes it as an illness, its immune response activation time is shortened by not requiring a raise in body temperature for the white blood T-cell to takeout the virus in the respiratory path. In another word, the body has build-up a natural resistance. Meaning it provides us an immune enhancing mechanism for patients by just having a blood infusion, with the like blood type obtained from COVID-19 100\% recovered patients. This process is safe and needs no long time drug testing. But simultaneously it also induces the natural evolution of the virus into another type of Coronavirus, such as SARS. We estimate that the $T_{c}$ for all coronaviruses lie between $110-130^{\circ} \mathrm{F}$. With MERS and SARS near the upper limit, COVID-19 is somewhat lower. That means its transmission is reduced in the tropics. Perhaps, we may interpret the current COVID-19 as an evolved form of SARS? And will spread faster. As such, our medication for SARS might be effective also on COVID-19? Furthermore, because for the virus to multiply and survive in the body, its spikes need to be attached to the respiratory path wall, a medication that suppresses that mechanism might also work? That includes medication used for HIV, Malaria and certain antibiotics which raises the virus formation temperature $T_{\mathcal{O}}$ as some bacterial infection requires that same attachment mechanism to harm the body's cell in the patient. During the early stage of the infection constant drinking of fluid can reduce the virus number along the respiratory upper path by washing the virus into the stomach, where it cannot survive. Therefore, as for medication, we might already have many well-tested drugs such as the Malaria drug helpful to cure COVID-19 for patients in the early stage only. But it is actually dangerous when used in patients already in the last stage, where the virus is destroying the lung. Of course for each of these drugs, testing must be made and effectiveness and side effects verified. As the northern hemisphere starts warming up due to approaching summer, we expect the COVID-19 spread to slow. Unfortunately, it also for the Southern Hemisphere to cool down as there autumn sets in, and we already observe the pandemic centers to shift there. Implying if we do not find a vaccine fast and soon, the pandemic cannot be stopped, and will surely again come back to the northern hemisphere later this year. On the bright side, if we have a vaccine, after this COVID-19 pandemic ends, the human race might for its new generation developed the necessary immune to it, or even new forms of Coronavirus that might come along affecting other mammal species? As to the pandemic's effect on the economy of the world, it will certainly put every country in a sharp depression, at least for $20 \%$ decrease in the GDP, or even more. No pandemic in human history has such an economic effect ever happened, even far more severe than the Spanish flu of 1919.

\section{Acknowledgements}

We thank Ms. Winnie So for her help in preparing the manuscript. 


\section{Conflicts of Interest}

The authors declare no conflicts of interest regarding the publication of this paper.

\section{References}

[1] Perelman, G. (2002) The Entropy Formula for the Ricci Flow and Its Geometric Application. arXiv.math.DG/0211159

Perelman, G. (2003) Ricci Flow with Surgery on Three-Manifolds.

arXiv.mathDG/0303109

[2] Wong, K.W., Fung, P.C.W. and Chow, W.K. (2020) Solar Radiation, Perelman Entropy Mapping, DNA, Viruses etc. Open Journal of Biophysics, 10, 54-58.

https://doi.org/10.4236/ojbiphy.2020.102005

[3] Ching, W.Y., Xu, Y.N., Zhao, G.L., Wong, K.W. and Zandiehnadem, F. (1987) Electronic Structure and Excitonic-Enhanced Superconducting Mechanism in $\mathrm{YBa}_{2} \mathrm{Cu}_{3} \mathrm{O}_{7-\delta}$, Physical Review Letters, 59, 1333-1336.

https://doi.org/10.1103/PhysRevLett.59.1333

[4] Wong, K.W., Fung, P.C.W. and Chow, W.K. (2019) 5D Model Theory for Creating of Life Forms. Journal of Modern Physics, 10, 1548-1565.

https://doi.org/10.4236/jmp.2019.1013103

[5] Wong, K.W. and Ching, C.W. (1988) Thermodynamics of Simultaneous-Excitonic Superconductivity Condensate. Physica C, 152, 397-400.

https://doi.org/10.1016/0921-4534(88)90043-3

[6] Wong, K.W. and Ching, C.W. (1989) The Theory of Simultaneous Excitonic-Superconductivity Condensation. Physica $C, 158,1-14$.

https://doi.org/10.1016/0921-4534(89)90294-3

[7] Maxmen, A. (2020) Slew of Trials Launch to Test Coronavirus Treatments in China. Nature, 578, 347-348. https://doi.org/10.1038/d41586-020-00444-3

[8] Richards, S.E. (2020) How Coronavirus Mutations Can Track Its Spread-And Disprove Conspiracies. National Geographic, Coronavirus Coverage. 